\title{
Disproportionate vitamin A deficiency in pregnant women of specific ethnicities in the United States and ethnic differences in allele frequencies of polymorphisms of vitamin A-related genes
}

\section{Masako Suzuki ( $\nabla$ masako.suzuki@einsteinmed.org )}

Albert Einstein College of Medicine https://orcid.org/0000-0003-0605-9225

\section{Tao Wang}

Albert Einstein College of Medicine

Diana J Garretto

Stony Brook University Hospital

Carmen R. Isasi

Albert Einstein College of Medicine

\section{Wellington V Cardoso}

Columbia University

John M. Greally

Albert Einstein College of Medicine

Loredana Quadro

Rutgers University

\section{Short report}

Keywords: Vitamin A deficiency, pregnant women, Hispanics, NHANES

Posted Date: January 29th, 2021

DOI: https://doi.org/10.21203/rs.3.rs-49188/v3

License: (c) (1) This work is licensed under a Creative Commons Attribution 4.0 International License. Read Full License 


\section{Abstract}

\section{Background}

Vitamin A is an essential micronutrient that plays critical roles in many biological functions of the body. The current national prevalence rate of vitamin A deficiency (VAD) in the United States is reported to be very low (<1\%). However, our recent study in an urban city of the U. S. (the Bronx study) revealed that pregnant women in the Bronx have much higher proportions of VAD than the national prevalence rate. Given that Hispanics (56\%) and non-Hispanic Blacks (29\%) are the major racial and ethnic groups in the Bronx, we hypothesized that VAD could be more prevalent among pregnant women from specific ethnicities in the U.S. We therefore re-analyzed two independent datasets of serum retinol levels, i.e., the data from the the Bronx study and the National Health and Nutrition Examination Survey (NHANES). Moreover, as known polymorphisms have been associated with vitamin A status, we also assessed the differences of minor allele frequencies of these polymorphisms between ethnic groups in publicly available datasets, such as the Allele Frequency Aggregator (ALFA), the Population Architecture using Genomics and Epidemiology (PAGE), and the 1000 Genomes project.

\section{Findings}

We found that in both datasets of pregnant women non-Hispanic Black and Hispanic ethnicities have high proportions of VAD compared to non-Hispanic White pregnant women, and this VAD prevalence rate was much higher of the currently estimated national prevalence level. Interestingly, non-Hispanic Black pregnant women showed comparably high proportions of VAD in both datasets. However, pregnant women with Latin American/Afro-Caribbean ancestry in the Bronx dataset have strikingly high proportion of VAD compared to Latin American/Mexican ancestry in NHANES dataset $(p=1.973 \mathrm{e}-10,95 \% \mathrm{Cl} 0.04$ 0.22 , Fisher's exact test). Furthermore, from the ALFA and the PAGE data analysis, we showed that the known single nucleotide polymorphism (SNP) located near the RBP4 gene (rs10882272) associated with lower serum retinol levels occurs at higher frequencies in Latin American/Afro-Caribbean ancestry and non-Hispanic Black/African populations compared to Latin American/Mexican ancestry and European populations. In addition, the analysis of minor allele frequency (MAF) of 39 previously reported SNPs associated with vitamin A metabolism showed significantly higher MAF variations between populations of different ancestries than that of randomly selected SNPs $(p=0.030$, permutation test with 1,000 iterations).

\section{Conclusions}

We confirmed that VAD rates in the pregnant women differ between different ethnicities, and that pregnant women in minority groups in the U.S. have much higher VAD rates than the estimated national prevalence level. Moreover, our analysis suggested that ethnic differences in allele frequencies of polymorphisms of vitamin A-related genes might contribute to the observed VAD rate differences. Further genome-wide association studies are needed to assess the influences of specific genetic variation and the different VAD status between different ethnic groups. 


\section{Main Text}

The essential micronutrient vitamin A plays critical roles in vision [1, 2], immune system [3, 4], cell growth and differentiation [5-8], as well as in the development of multiple organs, including the lung, heart, eyes, and kidneys (reviewed in $[9,10]$ ). Since vitamin $A$ is an essential micronutrient, limited access to vitamin A-rich food or supplements severely affects tissue and blood levels of vitamin $A$ in humans [11]. While the current prevalence rate of vitamin A deficiency (VAD) - defined as serum retinol levels lower than 1.05 $\mu \mathrm{mol} / \mathrm{I}$ - is estimated to be less than $1 \%$ in the United States (U.S.) (CDC's Second Nutrition Report [12]), it has been suggested that this rate might vary between different ethnic groups and races [13]. Interestingly, an epidemiological study based on the NHANES dataset, collected from 2003 to 2006, demonstrated that non-Hispanic Black women of childbearing age have a higher rate of VAD [14]. However, this dataset was collected almost two decades ago. Morevoer, in general, populations-level studies of vitamin A are not up to date in developed countries, including the U.S. [15].

Recently, we reported a high VAD proportion rate among pregnant women in the Bronx, NY, USA [16], where the ethnic diversity and the poverty rate is much higher than in the rest of the nation $[17,18]$. Although the original study addressed the effects of bariatric surgery on serum vitamin A levels during pregnancy, the most surprising result was that more than $60 \%$ of the pregnant women who did not undergo the bariatric surgery (control group) had serum retinol levels during the third-trimester lower than $1.05 \mu \mathrm{mol} / \mathrm{l}$ [16], meeting the criteria for vitamin A deficiency. This proportion of vitamin A deficient women in the Bronx was much higher than that of non-White women of the same age group recently reported by Hanson et al. [19]. As Hispanics (56\%) and non-Hispanic Black (29\%) are the major race and ethnic groups in the Bronx [18], our findings [16] prompted us to re-analyze the disproportionality of VAD status in the Hispanic and non-Hispanic Black pregnant women from the Bronx study. Here we used Student's t-test for continuous variables, Fisher's exact test for categorical variables, and a permutation test to assess the significance level of the deviations of allele frequency of previously reported vitamin $A$ related gene polymorphisms between the ethnic groups compared to background noise. An alpha of 0.05 was used as the cutoff for significance. All statistical analyses were performed using R version 4.0.2 [20]. In the Bronx dataset, women planning to breasfeed with singleton pregnancy with and without a history of bariatric surgery (either Roux-en-Y or gastric sleeve) were recruited [16]. Demographic data included race/ethnicity, education, pre-pregnancy and at delivery body mass index, gestational weight gain and parity. Pregnancy outcome included gestational age at delivery, mode of delivery, and neonatal weight [16]. We used the third-trimester serum retinol levels that were available for 67 women out of the 96 participants in the Bronx study [16]. We found no significant association between missing data status and other covariates. While maternal serum levels of beta-carotene, the most abundant dietary vitamin $A$ precursor [11], and cord blood serum retinol were significantly associated with maternal VAD status $(p=0.041$ and $p=0.007$, respectively), other known covariates, including the bariatric surgery status, did not show significant associations (Table 1). We then re-analyzed the third trimester serum retinol levels in the Bronx dataset by ethnic group, specifically using the self-reported ethnicities (non-Hispanic Black, Hispanic, or other race) as the ethnicities [16]. Our results showed that the proportion of VAD in Hispanic women was $65.9 \%$ (29 out of 44 Hispanic participants), in non-Hispanic Blacks was $53.3 \%$ (8 of 15 
African American participants), and for other ethnicities was 37.5\% (3 in 8 participants). Among the Hispanic participants $(n=44)$, vitamin A deficient women tended to be younger than the vitamin $A$ sufficient women $(p=0.088)$, but education levels, pre-pregnancy body mass index $(B M I)$, and gestational weight gain (GWG) were not associated with the VAD status $(p=0.876, p=0.195$, and $p=0.935$, respectively). Whereas the poverty levels were not assessed in the Bronx study cohort [16], the degree of education and poverty level are generally negatively correlated in the Bronx, NY (poverty rate with educational attainment of less than High School is $36.6 \%$, High School is $23.4 \%$, and College is $19.5 \%$, 2019 1-year estimates, U.S. Census) [18]. Therefore, we inferred that the poverty levels in the Bronx study were likely similar between vitamin A deficient and sufficient women, and that the poverty level might not have been directly associated with VAD status between ethnicities, in this cohort. Also, no clinical evidence was available for these VAD pregnant females as they were not assessed [16]. Nevertheless, overt symptoms of VAD were not expected given their subclinical vitamin A deficient status (based on serum levels). Therefore, despite the relatively limited sample size of the Bronx study, our analysis revealed that the proportions of VAD in Hispanic and non-Hispanic Black pregnant women were much higher than the estimated levels in the U.S. regardless of the history of bariatric surgery (Additional file 1).

Next, we analyzed serum retinol levels of pregnant women from the NHANES dataset (2001-2002, 20032004, and 2005-2006) by ethnicities using the reported race/ethnicity (RIDRETH1) information in the Sample Person Demographics Files [21]. While the NHANES data were collected almost two decades ago, this remains the latest nationwide serum vitamin A level assessment in the U.S.. In the NHANES dataset the proportion of VAD among Hispanic (Mexican American) pregnant women was half that of nonHispanic Black pregnant women, $14.9 \%$ and $32.0 \%$ respectively $(p<0.0001$, Fisher's exact test, Additional file 1; 966 pregnant women). According to the U. S. Census Bureau the poverty income ratio (PIR) of 1.0 defines the minimum income needed to avoid poverty and thus it is used as measure of poverty threshold [18]. Blumberg et al. [22] reported that individuals in poverty income ratio (PIR ) $>1.85$ subgroup had a lower prevalence of inadequacy for most nutrients compared to the subgroups PIR $\leqq 1.85$ [22]. Given that in the Bronx about $45 \%$ of people live below PIR $\leqq 1.85$ [18], we focused our analysis of the NHANES dataset on pregnant women aged 17-42 (similar to the age range of the Bronx study) with a PIR less than 1.85. While VAD status and PIR $\leqq 1.85$ were significantly associated in non-Hispanic Black women in total (aged 17-42, Fisher's exact test $p<0.005,95$ percent confidence interval (95\% Cl) 1.37-4.45), this association was not observed in pregnant women of all races [Hispanic (Mexican American), Fisher's exact test $p=0.6494$ (95\% Cl 0.53-4.57); non-Hispanic Black, Fisher's exact test $p=0.12(95 \% \mathrm{Cl} 0.83-6.73)$; non-Hispanic White, $\mathrm{p}=0.684(95 \% \mathrm{Cl} 0.49-2.71)]$. Taken together these data suggest that the higher VAD rates among pregnant women between these minority groups (Hispanic and non-Hispanic Black) might be independent of their poverty levels also in NHANES data set. Interestingly, the proportions of VAD in non-Hispanic Black pregnant women were not significantly differ between the NHANES and the Bronx datasets $(\mathrm{p}=0.26,95 \% \mathrm{Cl}$ 0.14-1.74), however, the proportions of VAD in Hispanic (Mexican American) pregnant women in poverty from the NHANES dataset were significantly lower than that of Hispanic pregnant women in the Bronx dataset $(p=1.973 e-10,95 \% \mathrm{Cl} 0.04-0.22)$. Of note, while the major origins 
of Hispanic populations in the Bronx are Latin Americans with Afro-Caribbean ancestry (39.4\% Dominicans and 36.4\% Puerto Ricans, U.S. Census data [18]), the Hispanic participants of the NHANES were Mexican Americans. Thus, these data strongly argue for an ancestry/ethnicity-specific impact on serum retinol levels and vitamin A status of pregnant women.

Genetic contributions to the levels of circulating retinol have been reported in European populations. Specifically, a family study in France showed that the heritability estimate for serum retinol concentration $(30.5 \%)$ was much larger than the variability accounted for by household, i.e., individuals living in the same house (14.2\%) [23]. Moreover, in the GWAS Catalog [24], two single nucleotide polymorphisms, (SNPs) (rs10882272 T/C and rs1667255 C/A), located near the retinol binding protein $4(R B P 4)$ and transthyretin (TTR) genes, respectively, identified from a genome-wide association study of 5,006 "Caucasian" males, are listed as associated with serum retinol levels [25]. The association of rs10882272 was replicated in independent samples, including 3,792 females and 504 males [25]. However, as the rate of VAD in the individuals studied was low, the authors were not able to test the association of the genetic variation with VAD [25].

Unfortunately, genomic data are not available for the Bronx nor for the NHANES study. Therefore, to assess the potential genetic contribution to the vitamin A status, we analyzed the deviations of allele frequency of previously reported vitamin A-related gene polymorphisms [25-27] between ethnic groups from publicly available population allele frequency datasets, such as the Allele Frequency Aggregator (ALFA) [28], the Population Architecture using Genomics and Epidemiology (PAGE [29], BioProject Accession: PRJNA168052), and the 1000 Genomes [30] projects. The ALFA pipeline consists of the allele frequency for variants in $\mathrm{dbGaP}$ that includes genomic data with subjects from 12 diverse populations worldwide [28]. The PAGE consortium dataset includes genetic variations representing seven ethnic groups, i.e., African Americans, Asian Americans, American Indians, European Americans, Hispanic Americans, and Native Hawaiians, from the United States-based cohorts [29]. Importantly, the Hispanic American cohorts in PAGE contains subjects from the Hispanic Community Health Study/Study of Latinos (HCHS/SOL), with one of the HCHS/SOL research centers located in the Bronx, NY [29, 31]. The associations of genetic variants with gene expression levels, defining expression quantitative trait loci (eQTL), were obtained from the Genotype-Tissue Expression project, GTEx [32]. To assess if the two above-mentioned genetic polymorphisms could be associated with VAD status differences between different racial/ethnic groups, we first compared the low serum retinol allele frequencies of rs 10882272 and rs1667255 between different ethnic groups in ALFA and PAGE. While we did not observe significant differences in major allele frequencies of rs1667255 between Hispanic groups (Additional file 2), the allele frequencies of rs10882272 showed significant variation between different ethnic groups, as we predicted. The frequency of the allele associated with low serum retinol (rs10882272: frequency for $\mathrm{C}$ allele) was much higher in African (0.620,62\%) and African American (0.617) compared to European (0.383) and Asian (0.106) individuals in the ALFA dataset (Figure 1a). Similarly, the PAGE dataset results showed that the risk allele frequencies were higher in Latin Americans with Afro-Caribbean ancestry [Puerto Ricans (0.455), Dominicans (0.502) and Cubans (0.410)] compared to Mexicans (0.260), Central Americans (0.288), South Americans (0.278) or Native Americans (0.357) (Figure 1b). The ethnic groups 
with the higher VAD proportions showed a higher frequency of the allele associated with low serum retinol levels. The rs 10882272 variant is located in the $3^{\prime}$ UTR of the free fatty acid receptor 4 (FFAR4) gene and downstream of the RBP4 gene. The FFAR4 gene encodes a GPCR receptor (GPCR120) for free long-chain fatty acids, including omega-3 [33,34]. FFAR4/GPCR120 is expressed in various cell types, including pituitary, lung, macrophages, adipocytes, intestinal neuroendocrine cells and pancreatic cells. Thus, it participates in a number of physiological processes, including energy regulation, insulin sensitivity, immunological homeostasis and anti-inflammatory responses [35]. RBP4 is the sole specific carrier for retinol in the bloodstream [2,36]. Predominantly expressed in the hepatocytes, RBP4 binds retinol to mobilize vitamin A from the liver, the primary body storage site of the vitamin, towards the peripheral tissues [2]. Furthermore, we tested if the rs 10882272 is an expression quantitative trait locus (eQTL) for its nearby genes using a publicly available database (the Genotype-Tissue Expression project, GTEx) [32]. In the GTEx data, we detected the associations between the rs 10882272 variations and the expression levels of RBP4 in the liver, where the gene is highly expressed [37], with the presence of the allele associated with low serum retinol levels also associated with increased expression of RBP4 (normalized effect size: $0.137, \mathrm{p}=0.00012$, and $\mathrm{m}$-value 0.987 ). For FFAR4, we detected the association in lung (normalized effect size: $0.126, \mathrm{p}=8.5 \mathrm{e}-6$, and $\mathrm{m}$-value 1.00 ), but not in pituitary (normalized effect size: $0.0334, p=0.5$, and $m$-value 0.809 ). The pituitary showed the highest expression of FFAR4 in the GTEx data.

Another polymorphism associated with differences in serum retinol levels between different ethnic groups is rs738409 [27], which is located in the patatin-like phospholipase domain containing 3 (PNPLA3) gene. PNPLA3 encodes a gene involved in the mobilization of retinyl esters stored in stellate cells $[27,38]$. The rs738409 polymorphism is a missense variant, with the $C$ to $G$ nucleotide substitution changing the amino acid I[ATC] to M[ATG]. The PNPLA3 I148M missense variant is a loss-of-function mutation [27], and associations between the variant and the risk of nonalcoholic fatty liver disease (NAFLD) have been reported [39-41]. The frequency of the mutant allele varies between ethnic groups (African American (0.144), Cuban (0.28), Dominican (0.26), Mexican (0.50) and Puerto Rican (0.34); PAGE dataset, Additional file 2). Of note, individuals homozygous for PNPLA3 I148M have lower circulating levels of RBP4 [27]. Changes in circulating levels of RBP4 have been linked to pathological conditions and variations in nutritional intake [42-45]. Interestingly, reported associations of the circulating levels of RBP4 and NAFLD are conflicting, and a recent meta analysis reported that circulating RBP4 levels may indeed not be associated with NAFLD [46]. Thus, we speculate that the associations between PNPLA3 I148M variants and circulating RBP4 levels might be independent of NAFLD status. In animal models, while retinol deficiency leads to accumulation of RBP4 in liver, likely by inhibiting its secretion from this organ, hepatic RBP4 mRNA levels show no differences between vitamin A deficiency and sufficiency $[47,48]$. Further studies are needed to test the associations of these SNPs with circulating RBP4 levels, serum retinol levels, and disease status.

In addition to the three above-mentioned SNPs, several GWAS and candidate gene association studies have identified other polymorphisms associated with serum retinol and beta-carotene levels as well as with the beta-carotene bioactivities [26]. We therefore also assessed the allele frequency deviations of the 
39 SNPs associated with circulating vitamin A levels [26] between different ethnic groups in the 1000 Genomes Project [30]. The deviations of allele frequencies of those vitamin A-related SNPs between different ethnic groups are listed in the Additional file 2. The average of the allele frequency standard deviation among ethnic groups was 0.122 , significantly higher than randomly selected sets of 39 SNPs from the 1000 Genomes data ( $p=0.030$, permutation test with 1,000 iterations, Additional file 1$)$. Since serum retinol level variations between different ethnic groups have been reported, this result is not surprising. However, this is the first systematic analysis of the allele frequency variations of the vitamin Arelated SNPs among different ethnic groups.

Looker et al. reported serum retinol level differences among three Hispanic groups using the Hispanic Health and Nutrition Examination Survey (HHANES) conducted from 1982-1984 [49]. The authors found that Mexican Americans have a higher VAD prevalence rate than Puerto Ricans or Cubans in both adults and children. This study was performed almost four decades ago when the participants' nutrient status might have differed from currently. The CDC's Second Nutrition Report in 2012 showed that serum vitamin A concentrations increased between 1999-2000 and 2005-2006 in the United States (geometric mean, $52.8 \mu \mathrm{g} / \mathrm{dl}$ to $54.7 \mu \mathrm{g} / \mathrm{dl}$ ). Moreover, serum retinyl palmitate levels, an indicator of newly ingested vitamin $A$, were dramatically increased during this period in Mexican Americans (geometric mean 0.759 $\mathrm{ug} / \mathrm{dl}$ in 1999-2000 to $1.85 \mathrm{ug} / \mathrm{dl}$ in 2005-2006) [50]. The differences observed in 1982-1984 might reflect the lower dietary intake of vitamin A at that time rather than genetic variations. An updated populationbased measurement of serum retinol levels is clearly needed.

We acknowledge that there are several limitations to our study: the sample size of the Bronx cohort $(n=97)$ was limited, the poverty levels and clinical information on VAD-related outcome of the Bronx cohort are not available, the serum retinol data of NHANES were collected almost two decades ago, and the genotype information of all participants is not available in both cohorts. Further genome-wide association studies with demographic information, including food accessibility/intake in multiethnic cohorts, are needed to assess the influences of genetic variation and the different VAD status between different ethnic groups. While the WHO does not recommend routine vitamin A supplementation to pregnant women, they recommend vitamin A supplementation to pregnant women in a given geographical area if $\geq 20 \%$ of pregnant women have serum retinol levels $<0.70 \mu \mathrm{mol} / \mathrm{L}$ [51]. Our reanalysis of the Bronx study showed that more than $40 \%$ of pregnant women have serum retinol $<0.70$ $\mu \mathrm{mol} / \mathrm{L}$, strongly suggesting that urgent actions need to be taken to reduce the VAD, especially in unusually susceptible ethnic groups, to reduce the risk of adverse health conditions of the mother [52] and diseases of offspring later in life [53, 54].

In summary, while VAD in developed countries is believed to be a rare condition, there is a substantial proportion of VAD pregnant women of certain ethnic groups, even in wealthy, developed countries. Moreover, our results showed that genetic polymorphisms may be contributing to the VAD status differences between ethnic groups, at least in pregnant women. Further understanding of this association will ultimately enable adequate food interventions based on the genetic information that could be crucial to improve maternal vitamin A status during pregnancy in these higher risk groups. 


\section{Abbreviations}

eQTL: expression quantitative trait loci

GWAS: Genome-Wide Association Study

NHANES: National Health and Nutrition Examination Survey

PAGE: Population Architecture using Genomics and Epidemiology

VAD: vitamin A deficiency

\section{Declarations}

\section{Ethical Approval and Consent to Participate:}

We used publicly available data, no ethical approval is required.

\section{Consent for publication:}

Not applicable.

\section{Availability of data and materials:}

The serum retinol and demographic information of the Bronx dataset, originally published in the $\mathrm{J}$ Perinat Med (PMID:30231012), are available by request. The National Health and Nutrition Examination Survey (NHANES) datasets were downloaded from the NHANES repository (https://www.cdc.gov/nchs/nhanes/index.htm) and merged file in accordance with the NHANES guidelines and recommendations.

\section{Competing interests:}

All authors declare no competing interest.

\section{Funding:}

This work was supported by the National Institutes of Health under award number R01HL145302 (MS) and partially by NIH R01HD094778 and R01 HD083331 (LQ). The content is solely the responsibility of the authors and does not necessarily represent the official views of the National Institutes of Health.

\section{Authors' Contributions:}

Wrote manuscript draft (MS,LQ), prepared illustrations (MS), approved final manuscript (MS, DG, TW, CRI, WC, JMG, LQ), conceived project (MS, LQ), analyzed data (MS), formulated research questions (MS, LQ), 
interpreted results (MS, TW, DG, CRI, JMG, WC, LQ), led investigation (MS). All authors read and approved the final manuscript.

\section{Acknowledgements:}

Not applicable.

\section{Authors' information:}

Department of Genetics, Albert Einstein College of Medicine, Bronx, NY, USA

John M. Greally and Masako Suzuki

Department of Epidemiology and Population Health, Albert Einstein College of Medicine, Bronx, NY, USA Tao Wang and Carmen R. Isasi

Department of Obstetrics and Gynecology and Women's Health, Stony Brook University Medical Center, Stony Brook, NY, USA

Diana Garretto

Department of Medicine, Columbia University, New York, NY USA

Wellington V. Cardoso

Department of Food Science and Rutgers Center for Lipid Research, and New Jersey Institute for Food, Nutrition, and Health, Rutgers University, New Brunswick, NJ, USA

Loredana Quadro

\section{References}

1. Vogel S, Piantedosi R, O'Byrne SM, Kako Y, Quadro L, Gottesman ME, et al. Retinol-binding proteindeficient mice: biochemical basis for impaired vision. Biochemistry. 2002;41:15360-8. doi:10.1021/bi0268551.

2. Quadro L, Blaner WS, Salchow DJ, Vogel S, Piantedosi R, Gouras P, et al. Impaired retinal function and vitamin A availability in mice lacking retinol-binding protein. EMBO J. 1999;18:4633-44. doi:10.1093/emboj/18.17.4633.

3. Liu X, Cui T, Li Y, Wang Y, Wang Q, Li X, et al. Vitamin A supplementation in early life enhances the intestinal immune response of rats with gestational vitamin A deficiency by increasing the number of immune cells. PLoS One. 2014;9:e114934. doi:10.1371/journal.pone.0114934.

4. Sirisinha S. The pleiotropic role of vitamin A in regulating mucosal immunity. Asian Pac J Allergy Immunol. 2015;33:71-89. 
5. Williams JB, Napoli JL. Metabolism of retinoic acid and retinol during differentiation of F9 embryonal carcinoma cells. Proc Natl Acad Sci USA. 1985;82:4658-62. doi:10.1073/pnas.82.14.4658.

6. Lee TF, Mak KM, Rackovsky O, Lin Y-L, Kwong AJ, Loke JC, et al. Downregulation of hepatic stellate cell activation by retinol and palmitate mediated by adipose differentiation-related protein (ADRP). J Cell Physiol. 2010;223:648-57. doi:10.1002/jcp.22063.

7. Cammas L, Romand R, Fraulob V, Mura C, Dollé P. Expression of the murine retinol dehydrogenase 10 (Rdh10) gene correlates with many sites of retinoid signalling during embryogenesis and organ differentiation. Dev Dyn. 2007;236:2899-908. doi:10.1002/dvdy.21312.

8. Janick-Buckner D, Barua AB, Olson JA. Induction of HL-60 cell differentiation by water-soluble and nitrogen-containing conjugates of retinoic acid and retinol. FASEB J. 1991;5:320-5. doi:10.1096/fasebj.5.3.2001792.

9. Marx J, Naudé H, Pretorius E. The Effects of Hypo- and Hypervitaminosis a and Its Involvement in Foetal Nervous System Development and Post-Natal Sensorimotor Functioning - A Review. The British Journal of Development Disabilities. 2006;52:47-64. doi:10.1179/096979506799103677.

10. Zile MH. Vitamin A and embryonic development: an overview. J Nutr. 1998;128 2 Suppl:455S-458S. doi:10.1093/jn/128.2.455S.

11. Tanumihardjo SA, Russell RM, Stephensen CB, Gannon BM, Craft NE, Haskell MJ, et al. Biomarkers of Nutrition for Development (BOND)-Vitamin A Review. J Nutr. 2016;146:1816S-48S. doi:10.3945/jn.115.229708.

12. CDC. CDC's Second Nutrition Report:A comprehensive biochemical assessment of the nutrition status of the U.S. population. https://www.cdc.gov/nutritionreport/pdf/4page_\%202nd\%20nutrition\%20report_508_032912.pdf. Accessed 20 May 2020.

13. Schleicher RL, Sternberg MR, Pfeiffer CM. Race-ethnicity is a strong correlate of circulating fatsoluble nutrient concentrations in a representative sample of the U.S. population. J Nutr. 2013;143:966S-76S. doi:10.3945/jn.112.172965.

14. Hanson C, Lyden E, Abresch C, Anderson-Berry A. Serum retinol concentrations, race, and socioeconomic status in of women of childbearing age in the united states. Nutrients. 2016;8. doi:10.3390/nu8080508.

15. Wirth JP, Petry N, Tanumihardjo SA, Rogers LM, McLean E, Greig A, et al. Vitamin A Supplementation Programs and Country-Level Evidence of Vitamin A Deficiency. Nutrients. 2017;9. doi:10.3390/nu9030190.

16. Garretto D, Kim Y-K, Quadro L, Rhodas RR, Pimentel V, Crnosija NA, et al. Vitamin A and $\beta$-carotene in pregnant and breastfeeding post-bariatric women in an urban population. $\mathrm{J}$ Perinat Med. 2019;47:183-9. doi:10.1515/jpm-2018-0142.

17. Toprani A, Li W, Hadler JL. Trends in Mortality Disparities by Area-Based Poverty in New York City, 1990-2010. J Urban Health. 2016;93:538-50. doi:10.1007/s11524-016-0048-7. 
18. U.S. Census Bureau. US Census. https://data.census.gov/cedsci/. Accessed 13 Jul 2020.

19. Hanson C, Schumacher MV, Lyden E, Su D, Furtado J, Cammack R, et al. Fat-soluble vitamins A and E and health disparities in a cohort of pregnant women at delivery. J Nutr Sci. 2018;7:e14. doi:10.1017/jns.2018.5.

20. R Foundation for Statistical Computing RCT. R: A Language and Environment for Statistical Computing. https://www.r-project.org/. Accessed 1 Nov 2020.

21. NHANES. National Health and Nutrition Examination Surveys (NHANES) . Centers for Disease Control and Prevention (CDC). National Center for Health Statistics (NCHS). National Health and Nutrition Examination Survey Data. Hyattsville, MD: U.S. Department of Health and Human Services, Centers for Disease Control and Prevention. https://wwwn.cdc.gov/nchs/nhanes/Default.aspx. Accessed 12 Jul 2020.

22. Blumberg JB, Frei B, Fulgoni VL, Weaver CM, Zeisel SH. Contribution of dietary supplements to nutritional adequacy by socioeconomic subgroups in adults of the united states. Nutrients. 2017;10. doi:10.3390/nu10010004.

23. Gueguen S, Leroy P, Gueguen R, Siest G, Visvikis S, Herbeth B. Genetic and environmental contributions to serum retinol and alpha-tocopherol concentrations: the Stanislas Family Study. Am J Clin Nutr. 2005;81:1034-44. doi:10.1093/ajcn/81.5.1034.

24. The NHGRI-EBI Catalog of human genome-wide association studies. GWAS Catalog. https://www.ebi.ac.uk/gwas/. Accessed 13 Jul 2020.

25. Mondul AM, Yu K, Wheeler W, Zhang H, Weinstein SJ, Major JM, et al. Genome-wide association study of circulating retinol levels. Hum Mol Genet. 2011;20:4724-31. doi:10.1093/hmg/ddr387.

26. Borel P, Desmarchelier C. Genetic Variations Associated with Vitamin A Status and Vitamin A Bioavailability. Nutrients. 2017;9. doi:10.3390/nu9030246.

27. Pirazzi C, Valenti L, Motta BM, Pingitore P, Hedfalk K, Mancina RM, et al. PNPLA3 has retinylpalmitate lipase activity in human hepatic stellate cells. Hum Mol Genet. 2014;23:4077-85. doi:10.1093/hmg/ddu121.

28. Allele Frequency Aggregator. https://www.ncbi.nlm.nih.gov/snp/docs/gsr/alfa/. Accessed 13 Jul 2020.

29. Population Architecture using Genomics and Epidemiology. https://www.pagestudy.org/index.php/home. Accessed 12 Jul 2020.

30. 1000 Genomes Project Consortium, Abecasis GR, Auton A, Brooks LD, DePristo MA, Durbin RM, et al. An integrated map of genetic variation from 1,092 human genomes. Nature. 2012;491:56-65. doi:10.1038/nature11632.

31. Sorlie PD, Avilés-Santa LM, Wassertheil-Smoller S, Kaplan RC, Daviglus ML, Giachello AL, et al. Design and implementation of the Hispanic Community Health Study/Study of Latinos. Ann Epidemiol. 2010;20:629-41. doi:10.1016/j.annepidem.2010.03.015.

32. GTEx Consortium. Human genomics. The Genotype-Tissue Expression (GTEx) pilot analysis: multitissue gene regulation in humans. Science. 2015;348:648-60. doi:10.1126/science.1262110. 
33. Oh DY, Talukdar S, Bae EJ, Imamura T, Morinaga H, Fan W, et al. GPR120 is an omega-3 fatty acid receptor mediating potent anti-inflammatory and insulin-sensitizing effects. Cell. 2010;142:687-98. doi:10.1016/j.cell.2010.07.041.

34. Hirasawa A, Tsumaya K, Awaji T, Katsuma S, Adachi T, Yamada M, et al. Free fatty acids regulate gut incretin glucagon-like peptide-1 secretion through GPR120. Nat Med. 2005;11:90-4. doi:10.1038/nm1168.

35. Oh DY, Walenta E. Omega-3 Fatty Acids and FFAR4. Front Endocrinol (Lausanne). 2014;5:115. doi:10.3389/fendo.2014.00115.

36. Quadro L, Hamberger L, Colantuoni V, Gottesman ME, Blaner WS. Understanding the physiological role of retinol-binding protein in vitamin A metabolism using transgenic and knockout mouse models. Mol Aspects Med. 2003;24:421-30. doi:10.1016/s0098-2997(03)00038-4.

37. Thompson SJ, Sargsyan A, Lee S-A, Yuen JJ, Cai J, Smalling R, et al. Hepatocytes are the principal source of circulating RBP4 in mice. Diabetes. 2017;66:58-63. doi:10.2337/db16-0286.

38. Kovarova M, Königsrainer I, Königsrainer A, Machicao F, Häring H-U, Schleicher E, et al. The Genetic Variant I148M in PNPLA3 Is Associated With Increased Hepatic Retinyl-Palmitate Storage in Humans. J Clin Endocrinol Metab. 2015;100:E1568-74. doi:10.1210/jc.2015-2978.

39. Tian C, Stokowski RP, Kershenobich D, Ballinger DG, Hinds DA. Variant in PNPLA3 is associated with alcoholic liver disease. Nat Genet. 2010;42:21-3. doi:10.1038/ng.488.

40. He S, McPhaul C, Li JZ, Garuti R, Kinch L, Grishin NV, et al. A sequence variation (I148M) in PNPLA3 associated with nonalcoholic fatty liver disease disrupts triglyceride hydrolysis. J Biol Chem. 2010;285:6706-15. doi:10.1074/jbc.M109.064501.

41. Romeo S, Kozlitina J, Xing C, Pertsemlidis A, Cox D, Pennacchio LA, et al. Genetic variation in PNPLA3 confers susceptibility to nonalcoholic fatty liver disease. Nat Genet. 2008;40:1461-5. doi:10.1038/ng.257.

42. Graham TE, Yang Q, Blüher M, Hammarstedt A, Ciaraldi TP, Henry RR, et al. Retinol-binding protein 4 and insulin resistance in lean, obese, and diabetic subjects. N Engl J Med. 2006;354:2552-63. doi:10.1056/NEJMoa054862.

43. Manolescu D-C, Sima A, Bhat PV. All-trans retinoic acid lowers serum retinol-binding protein 4 concentrations and increases insulin sensitivity in diabetic mice. J Nutr. 2010;140:311-6. doi:10.3945/jn.109.115147.

44. Aeberli I, Biebinger R, Lehmann R, L'allemand D, Spinas GA, Zimmermann MB. Serum retinol-binding protein 4 concentration and its ratio to serum retinol are associated with obesity and metabolic syndrome components in children. J Clin Endocrinol Metab. 2007;92:4359-65. doi:10.1210/jc.20070468.

45. Jing L, Xiao M, Dong H, Lin J, Chen G, Ling W, et al. Serum Carotenoids Are Inversely Associated with RBP4 and Other Inflammatory Markers in Middle-Aged and Elderly Adults. Nutrients. 2018;10. doi:10.3390/nu10030260. 
46. Zhou Z, Chen H, Ju H, Sun M. Circulating retinol binding protein 4 levels in nonalcoholic fatty liver disease: a systematic review and meta-analysis. Lipids Health Dis. 2017;16:180. doi:10.1186/s12944-017-0566-7.

47. Blaner WS. Retinol-binding protein: the serum transport protein for vitamin A. Endocr Rev. 1989;10:308-16. doi:10.1210/edrv-10-3-308.

48. Soprano DR, Soprano KJ, Goodman DS. Retinol-binding protein messenger RNA levels in the liver and in extrahepatic tissues of the rat. J Lipid Res. 1986;27:166-71.

49. Looker AC, Johnson CL, Underwood BA. Serum retinol levels of persons aged 4-74 years from three Hispanic groups. Am J Clin Nutr. 1988;48:1490-6. doi:10.1093/ajcn/48.6.1490.

50. Centers for Disease Control and Prevention. Second Nutrition Report (2012). https://www.cdc.gov/nutritionreport/report_2012.html. Accessed 13 Jul 2020.

51. Vitamin A supplementation during pregnancy. https://www.who.int/elena/titles/guidance_summaries/vitamina_pregnancy/en/. Accessed $13 \mathrm{Jul}$ 2020.

52. Radhika MS, Bhaskaram P, Balakrishna N, Ramalakshmi BA, Devi S, Kumar BS. Effects of vitamin A deficiency during pregnancy on maternal and child health. BJOG. 2002;109:689-93. doi:10.1111/j.1471-0528.2002.01010.x.

53. Bhat PV, Manolescu D-C. Role of vitamin A in determining nephron mass and possible relationship to hypertension. J Nutr. 2008;138:1407-10. doi:10.1093/jn/138.8.1407.

54. Chen F, Marquez H, Kim Y-K, Qian J, Shao F, Fine A, et al. Prenatal retinoid deficiency leads to airway hyperresponsiveness in adult mice. J Clin Invest. 2014;124:801-11. doi:10.1172/JCI70291.

\section{Table 1}

Due to technical limitations Table 1 is available as a download in the Supplementary Files.

\section{Figures}


a

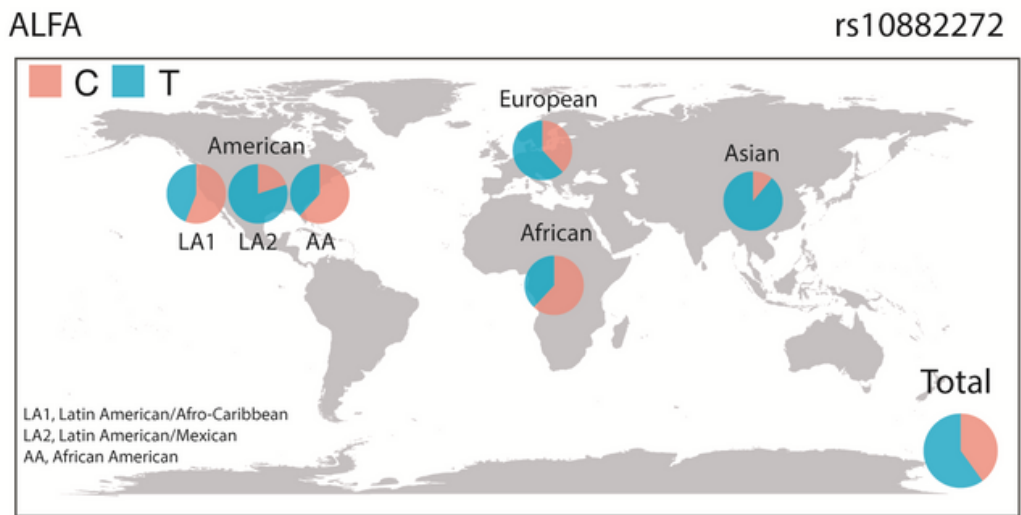

b

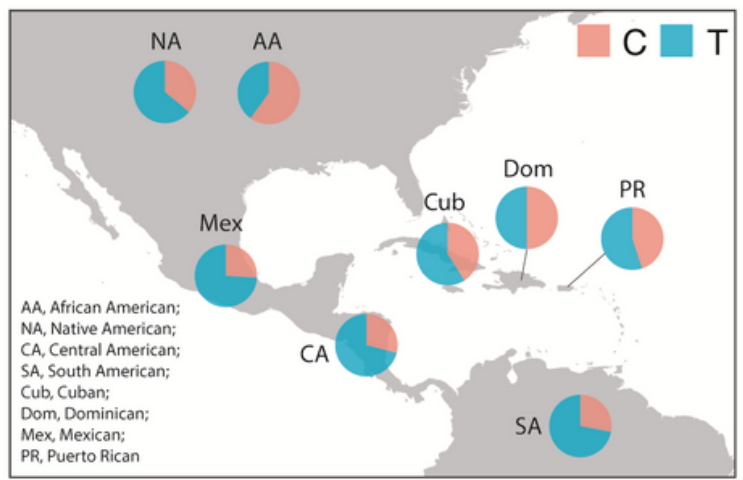

Figure 1

\section{Figure 1}

Variations of the allele frequencies of rs 10882272 We plotted the allele frequency of each ethnic group (a) Allele Frequency Aggregator (ALFA) and (b) Population Architecture using Genomics and Epidemiology (PAGE). Note: The designations employed and the presentation of the material on this map do not imply the expression of any opinion whatsoever on the part of Research Square concerning the legal status of any country, territory, city or area or of its authorities, or concerning the delimitation of its frontiers or boundaries. This map has been provided by the authors.

\section{Supplementary Files}

This is a list of supplementary files associated with this preprint. Click to download.

- Table1.xlsx

- Additionalfile1.pdf

- Additionalfile2.xlsx 